\title{
The effects of familiarity and practice on naming pictures of objects*
}

\author{
D. J. BARTRAM \\ Laboratory of Experimental Psychology, University of Sussex, Falmar, Brighton, Sussex, England
}

\begin{abstract}
Ss were given a paired-associate learning task, using nonsense shapes as stimuli and object names which varied in Thorndike-Lorge frequency as responses. Between each block of learning trials, Ss named the nonsense shapes and a set of line drawings of objects. While naming latencies for the shapes were unaffected by name frequency, there was an effect of frequency on naming a control set of pictures of objects. The frequency effect for the pictures decreased significantly with practice. When the Ss were asked to name pictures of the objects having the names previously learned for the nonsense shapes, an effect of frequency appeared, the size of the effect being the same as that found for the control pictures after practice. The frequency effect disappeared when the shapes were reintroduced.
\end{abstract}

It has been shown that the speed with which line drawings of objects can be named increases as an inverse function of the Thorndike-Lorge frequency of the objects' names (Oldfield \& Wingfield, 1964, 1965). Wingfield (1966, Experiment 3) has shown that the size of this "frequency effect" (the difference between naming latencies for common and rare objects) decreases with practice. In the experiment, a set of 26 pictures was named by 12 Ss, 4 of whom each returned for further naming sessions on two occasions within a week of the first naming session and again after periods ranging from 3 to 6 months. In the final session, they named the objects a total of 10 times (each naming block being followed by a $10-\mathrm{min}$ interval). The variability in interblock interval, both overall and between Ss, together with the small number of Ss, make it difficult to draw any clear conclusions. Clearly, this interaction between frequency and practice needs to be examined under more carefully controlled conditions.

Wingfield (1966, Experiment 8) has also shown that if Ss are given a task in which they encounter a set of object names (in this case, a free recall task), naming latencies for those objects are faster than for objects whose names are not experienced. However, 50 free recall trials have less effect on reducing naming latencies for an object than does one picture-naming trial. He argues that if an object's name is stored in the same location as information used in the process of perceptual identification, then rehearsing the name of the object should have the same effect on a subsequent naming task as actually naming the object. If, on the other hand, naming involves a separate name-retrieval stage after perceptual identification has occurred, complete transfer from the free recall task would not be expected. Thus,

\footnotetext{
*This work was carried out during the tenure of an SRC Research Studentship. The author is grateful to N. S. Sutherland and A. Baddeley for advice and discussion. Present address: Department of Psychology, University of Hull, Hull, Yorkshire, England.
}

the results of the experiment are taken to support the second "two-stage" hypothesis. However, it is not clear why retrieval of a name should necessarily involve retrieval of other information about the object that that name refers to, even if the name is stored in a similar location. It seems more likely that the amount of object information retrieved will depend on the requirements of the task. In a free recall task, it is not necessary to access as much information about the semantic and perceptual properties of the objects whose names are being learned as it is in a naming task. Furthermore, it is impossible to say how many free recall trials are equivalent to one naming trial, as the two types of tasks are so different.

In order to distinguish between the two hypotheses proposed by Wingfield (the "single-stage" and "two-stage" models), it is necessary to use a training task which is as similar to a naming task as possible but which does not actually involve naming pictures of objects. In the following experiment, Ss were given experience with a set of names by learning them as names for nonsense shapes. They then had to name these shapes as rapidly as possible. Each $\mathrm{S}$ was also given an equivalent amount of practice naming pictures of objects. Given that the nonsense-shape task is similar to an object-naming task and that Ss know that they will subsequently have to name the objects whose names they learn for the nonsense shapes, then when these objects are substituted for the nonsense shapes, the "single-stage" hypothesis predicts that naming latencies for the substituted objects will be the same as those for the practiced set of objects. The "two-stage" hypothesis predicts that, while some transfer would be expected, naming latencies for the substituted objects should be longer than for the practiced ones.

The use of a nonsense-shape naming task also makes it possible to assess the effects of a priori word frequency on response latencies. If the frequency effect is a function of name-retrieval processes which are independent of processes involved in perceptual 
Table 1

Experimental Design for One Subgroup of Three Ss*

\begin{tabular}{|c|c|c|c|}
\hline 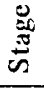 & $\begin{array}{l}\text { Naming } \\
\text { Blocks }\end{array}$ & $\begin{array}{c}\text { Objects } \\
\text { Stimulus-Response }\end{array}$ & $\begin{array}{l}\text { Nonsense-Shapes } \\
\text { Stimulus-Response }\end{array}$ \\
\hline 1 & 1 & Picture (P1)-Name (N1) & Shape (A)-"No" \\
\hline & $2-7$ & Picture (P1)-Name (N1) & Shape (A)-Name (N2) \\
\hline 2 & $8-10$ & Picture (P2)-Name (N2) & Shape (B)-"No" \\
\hline 3 & 11 & Picture (P1)-Name (N1) & Shape (A)-Name (N2) \\
\hline
\end{tabular}

*O1'erall, six Ss had Sets P1-N1 on Stage 1, half of them learning Set $N 2$ to Shape-Set $A$ and half to Shape-Set $B$, while the rest had Sets P2-N2, with half learning Set N1 to Shape-Set $A$ and half to Shape-Set $B$.

identification, effects of name frequency on nonsense-shape naming latencies should occur. On the other hand, it is more likely that the frequency effect results either from differences in the times taken to perceptually identify objects or, as proposed by Oldfield (1966), from differences in the times taken to retrieve the objects' names from memory. While the latter model holds that the frequency effect is a function of response retrieval processes, these processes are dependent upon a prior judgment as to the familiarity of the stimulus presented. Thus, naming equally familiar (or unfamiliar) nonsense shapes would not be expected to produce a frequency effect even when their names vary in frequency.

The present experiment investigates the way in which practice reduces naming latencies for objects of different degrees of familiarity. It also compares the effects of practice with naming a set of nonsense shapes and practice with naming a set of object pictures on a subsequent object-naming task. The experiment was carried out both to provide some information on practice effects in a naming task and to clarify certain of Wingfield's findings. The experiment was divided into three stages. In Stage 1, Ss learned names for a set of nonsense shapes and were given practice naming both the shapes and a control set of object pictures. In Stage 2, object pictures were substituted for the shapes (the names being kept the same), while in Stage 3, the conditions prevailing in Stage 1 were returned to.

\section{METHOD}

\section{Subjects}

Twelve undergraduates, three female and nine male, none of whom were psychology majors, were paid for their participation in a 1 -h session. They were tested individually.

\section{Materials}

Twenty objects, whose names varied in frequency from less than one per million to more than 100 (Thorndike \& Lorge, 1944), were represented by 20 line drawings (black on a plain white background). Objects were chosen that did not have alternative names and whose names were approximately equal in length. Two (banjo, window) were used for practice. Of the rest, nine (cello, trowel, guitar, snail, bugle, piano, hammer, rabbit, horse) were assigned to Picture Set P1 and their names to
Set $\mathrm{N} 1$, while the remainder (pliers, zebra, anvil, violin, camel, tiger, saddle, clock, chair) were assigned to Picture Set P2 and their names to Set $\mathrm{N} 2$. The frequency range covered by each set was balanced. Eighteen nonsense shapes having association values between $38 \%$ and $42 \%$ (Vanderplas \& Garvin, 1959) were selected such that they were all readily discriminable from one another. Using $\mathrm{P} / \sqrt{\mathrm{A}}(\mathrm{P}=$ perimeter, $\mathrm{A}=$ area) as a measure of complexity (Attneave, 1957), two sets (A and B), each of nine shapes, were produced such that the mean association value and complexity of each set were equal. Both object and nonsense-shape stimuli were prepared as $15 \times 10 \mathrm{~cm}$ cards on which both the stimulus and its name appeared and as $35-\mathrm{mm}$ slides on which just the stimulus appeared. The slides were back-projected onto a $14 \times 9 \mathrm{~cm}$ screen from a Kodak Carousel projector fitted with a solenoid-operated shutter and Polaroid-filter brightness control. The image filled the screen, and $S$ was seated $150 \mathrm{~cm}$ away.

\section{Experimental Design}

The two name sets, $\mathrm{N} 1$ and $\mathrm{N} 2$, were combined with the two shape sets, $A$ and $B$, to produce four paired-associate lists ( $A-N 1$, A-N2, B-N1, and B-N2), each consisting of nine pairs. Three Ss learned each list. The overall design of the experiment is presented in Table 1 for Ss assigned to List A-N2.

\section{Stage 1}

On the first naming block of Stage 1, Ss named pictures from one of the two picture sets and responded with the word "no" to the shapes from one of the shape sets. Each naming block consisted of nine shapes and nine pictures presented alternately. The two orders (picture-shape-picture-shape..., and shape-picture-shape-picture...) were balanced across Ss. Preceding each of the remaining six naming blocks, Ss were given a learning block in which they were taught names for the shapes (the names used being from Set $\mathrm{N} 1$ if Ss were naming pictures from Set $\mathrm{P} 2$ and from Set N2 if they were naming pictures from Set P1). Each learning block was carried out by presenting the 18 object names and stimuli ( 9 objects and 9 shapes) on the $15 x$ $10 \mathrm{~cm}$ cards. The Ss were instructed to read aloud the names written beneath the shapes and the objects. Each card was presented for $4 \mathrm{sec}$, with a 2 -sec interval between cards. Following each learning block, Ss had a naming block in which they had to name the objects and also try to name the nonsense shapes. No further learning blocks were given after Stage 1 .

\section{Stage 2}

Three naming blocks were given in which Ss named pictures from the other picture set (the set whose names they had learned for the shapes during Stage 1) and responded "no" to filler items from the other shape set.

\section{Stage 3}

This was a return to the conditions prevailing in Stage 1. One naming block was given with the original set of shapes and objects. It was not preceded by a learning block.

\section{Procedure}

Each naming trial was preceded by a .5 -sec warning light Naming latencies were recorded by means of a voice key, operation of which also terminated the stimulus exposure. In order to familiarize Ss with the apparatus, they were first given 16 trials, half with a blank field and the remainder with the letter "X." Ss had to respond "no" to the former and "yes" to the latter. Next, Ss were told that line drawings of objects would appear on the screen and that they were to name them as rapidly as possible. Having named the two practice stimuli, the design of 
the experiment was explained to them and the need for speed and accuracy of response on the naming trials was stressed. For the nonsense shapes, they were told to respond "no" on the first naming block and also on subsequent naming blocks if they did not know a shape's name. Prior to Stage 2 , they were reminded that they would see pictures of the objects whose names they had been learning for the shapes and that a new set of shapes would appear to which they should respond "no." Prior to Stage 3, they were told that they would again have the original set of names and shapes to respond to. Throughout the experiment, Ss were given immediate correction if they made errors on either object- or shape-naming conditions. A part from the constraint that picture and shape stimuli appear in a regular alternating order, they were otherwise randomly arranged within blocks. There was a short interval between each naming block while stimulus orders were changed.

\section{RESULTS}

\section{Errors}

For the object stimuli, all errors occurred on Naming Block 1 (an error rate of $17.6 \%$ for that block, $1.6 \%$ overall). Only 2 of the 19 errors made were not immediately corrected by $\mathrm{Ss}$ themselves. For the nonsense shapes, all Ss had reached $100 \%$ correct by the sixth naming block. As expected, no significant effect of either name set (N1 vs N2) or shape set (A vs B) was found on either rate of learning or naming speed. Also, there were no errors on Stage 3, even though no learning block was given during it.

\section{Naming Latencies}

The mean naming latencies for objects and shapes are presented in Fig. 1. A clear practice effect exists in both cases, though there is an increase in object-naming

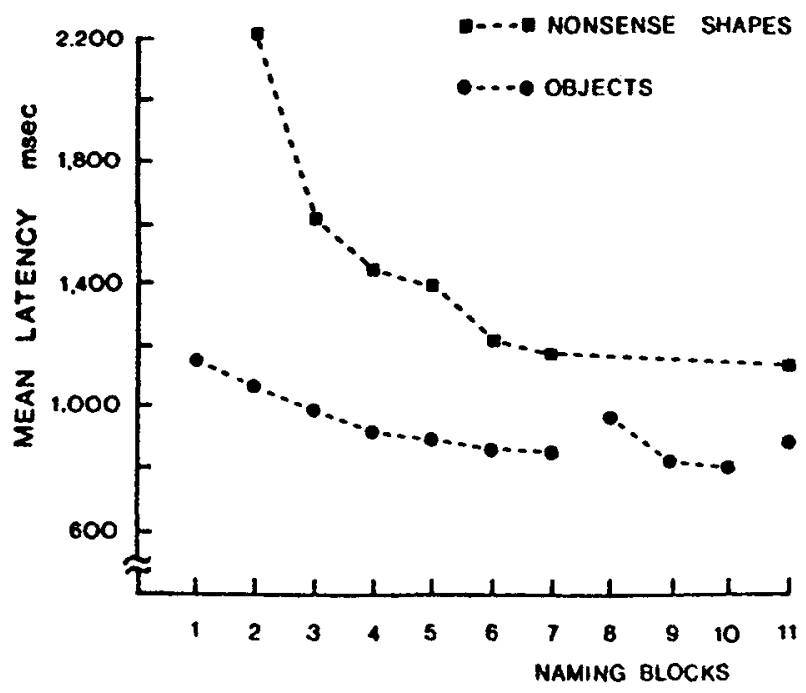

Fig. 1. The effects of practice on naming latencies for objects and nonsense shapes. For the objects, $N=108$, while for the nonsense shapes, $64 \leqslant N \leqslant 108$.

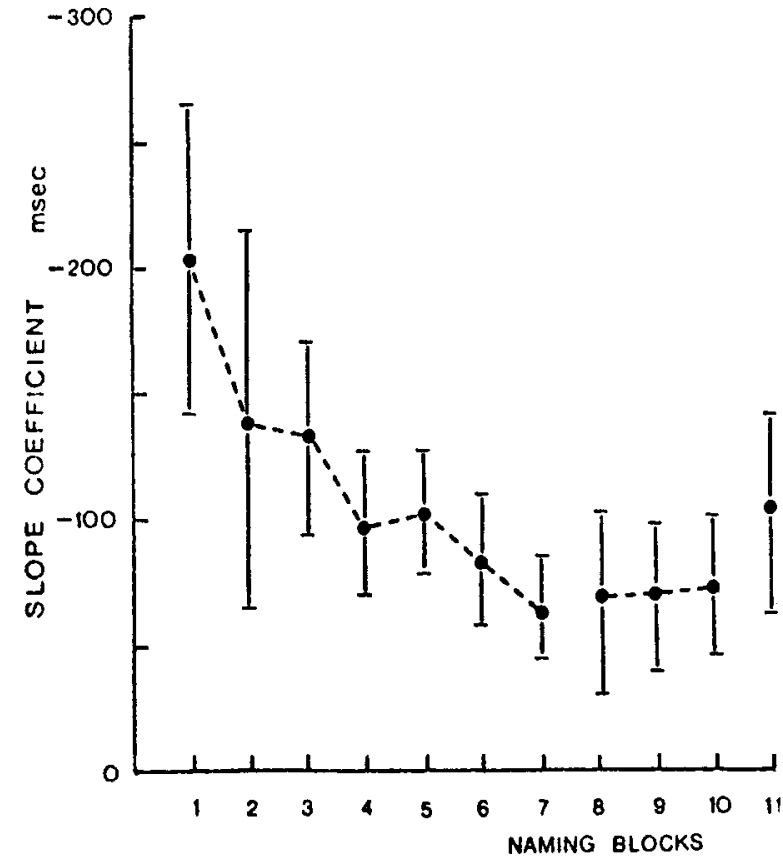

Fig. 2. Variations in the frequency effect. The slope coefficients and their standard errors of the linear regression equations relating object-naming latencies and frequency (milliseconds per log unit increase in frequency) for each block.

latencies after changing from Stage 1 to Stage 2 and from Stage 2 to Stage 3. Analyses of variance and regression analyses were carried out on the nonsense-shape naming latencies for Blocks 6, 7, and 11 . Contrary to the prediction of the stimulus-independent response retrieval model, there is no effect of name frequency on these latencies: for the last block of Stage 1 (Block 7), the slope of the function relating latency and frequency was $-4.2 \mathrm{msec}$ per $\log$ unit increase in frequency for the nonsense shapes $(F<1)$, while for the object stimuli it was $-64.49 \mathrm{msec}$ per log unit increase in frequency $[F(1,107)=10, p<.01]$. Again, for Stage 3, the effect of frequency was not significant for the nonsense shapes $(F<1)$ but was for the objects, with $-102.75 \mathrm{msec}$ per log unit increase in frequency $[F(1,107)=7, p<.05]$. The slope coefficients and their SEs for each block of object naming are presented in Fig. 2. Though the effect of frequency decreases with practice, it remains significant throughout the experiment for the object stimuli.

A more detailed examination of the effect of practice on naming latencies for objects was carried out by splitting the results for the 18 object stimuli into two sets [one of 6 low-frequency (LF) stimuli and one of 6 high-frequency (HF) ones], the middle 6 being discarded. The means and SDs for the LF and HF latencies are presented in Table 2. All the statistics that follow were carried out on these dichotomized data. For Stage 1, it was found that the effect of frequency [with $F(1,154)=136, p<.001]$ and the effect of practice 
Table 2

Low and High Frequency Means and SDs (Milliseconds)

\begin{tabular}{ccccccc} 
& \multirow{2}{*}{$\begin{array}{c}\text { Naming } \\
\text { Stage }\end{array}$} & \multicolumn{2}{c}{ Low Frequency } & & \multicolumn{2}{c}{ High Frequency } \\
1 & Blocks & Mean & SD & & Mean & SD \\
\hline & 1 & 1310 & 297 & 957 & 207 \\
& 2 & 1156 & 392 & 899 & 170 \\
& 3 & 1084 & 292 & 841 & 108 \\
& 4 & 1003 & 186 & 808 & 108 \\
& 5 & 988 & 207 & 803 & 99 \\
& 6 & 928 & 180 & 782 & 106 \\
& 7 & 907 & 136 & 802 & 87 \\
2 & 8 & 1033 & 328 & 896 & 162 \\
& 9 & 905 & 185 & 774 & 90 \\
3 & 10 & 871 & 238 & 766 & 135 \\
& 11 & 979 & 254 & 789 & 86 \\
\hline
\end{tabular}

[with $F(6,154)=18, p<.01]$ were significant and that the decrease in the size of the frequency effect associated with practice was also significant [with $\mathrm{F}(6,154)=2.9, \mathrm{p}<.05]$. The decrease in frequency effect across Stage 1 (between Blocks 1 and 7) was highly significant [with $t(22)=5.9, p<.005]$. The increase in frequency effect between Stages 1 and 2 was not significant, though that between Stages 2 and 3 was [with $t(22)=2.07, p<.05$ ]. This latter increase can be seen, from Table 2, to be due to LF latencies increasing from Stage 2 to Stage 3, while HF ones remain constant.

\section{DISCUSSION}

The results can be summarized as follows. The size of the frequency effect associated with object-naming latencies decreased significantly with practice. There was no effect of name frequency on the speed with which nonsense shapes were named, though there was an effect on object-naming latencies after an equivalent amount of practice (Stage 1). When the responses that were given to the nonsense shapes were subsequently used to name their respective object pictures, a significant effect of frequency was found (Stage 2). However, the size of this effect did not differ significantly from that found at the end of practice for object pictures in Stage 1. While the frequency effect did not increase from Stage 1 to Stage 2, there was an overall rise in naming latencies of about $100 \mathrm{msec}$ between the end of Stage 1 (Naming Block 7) and the beginning of Stage 2 (Block 8). By the second naming block of Stage 2 (Block 9), latencies were at the level found at the end of Stage 1. When nonsense shapes were again introduced, this frequency effect disappeared (Stage 3). After the interpolated naming task of Stage 2, naming latencies for LF objects were found to be greater in Stage 3 than they had been at the end of Stage 1. This did not apply to the HF objects.

As outlined above, the results appear to support the "single-stage" hypothesis, since the object-naming frequency effect was the same following practice with naming nonsense shapes as it was after naming pictures. While the frequency effects found at the beginning of
Stage 2 and the end of Stage 1 were equal, overall latencies were some $100 \mathrm{msec}$ longer. This increase may reflect the effects of stimulus uncertainty. Although the Ss knew at the beginning of Stage 2 the names of the objects they were to see, they did not know exactly what the pictures would look like. Once the set of pictures had been seen (Block 8), latencies dropped to the level associated with practiced $S$ s in Stage 1.

The fact that no frequency effect occurred for nonsense-shape naming is consistent with Oldfield's (1966) hypothesis that response retrieval processes are dependent upon some perceptual judgment about the familiarity of the object depicted. However, it is difficult to see how this two-stage model would predict the small frequency effect found for picture naming after training with nonsense shapes. Oldfield's theory holds that names of common objects are stored in relatively small "memory ensembles," while those of rare objects are stored in larger ensembles. In naming, the appropriate ensemble is selected, as a result of a decision about the familiarity of the depicted object, and a binary search of it is then carried out. Search time is a logarithmic function of ensemble size, and the whole of the frequency effect is attributed to differences in the times taken to search small HF ensembles and large LF ones. The effect of practice on reducing the frequency effect could be explained by arguing that practice has the effect of making the LF objects more "familiar," and so their names are transferred to smaller ensembles. As HF names are already in small ensembles, practice will have less effect on reducing latencies for these names than for the less common ones.

The transfer of the frequency effect from Stage 1 to Stage 2 can be explained if the following assumptions are made. First is that the learning of shape-name pairs is mediated by information about the objects referred to by the names. Second is that this retrieval of object information has the same effect, on transferring the names of LF objects to smaller ensembles, as does actual object naming. Third is that this mediation does not result in a frequency effect for nonsense-shape naming because the latencies are too long and variable to reveal any such effect. (This is possible, as the expected frequency effect would be in the order of only $150 \mathrm{msec}$ by the time Ss had reached criterion performance on Block 6).

It could also be argued that names are multiply represented in memory, a given name occurring in different-sized ensembles. Thus, the set of names would occur in one ensemble associated with the unfamiliar nonsense shapes and in others associated with the objects they refer to. If it is assumed that practice with a set of names affects all the representations of those names in memory, then practice with the nonsense shapes, while not producing a frequency effect, will have the same effect on names stored in object-related ensembles as practice with the objects themselves.

However, there is evidence to show that if 
photographs instead of line drawings are used as stimuli, the frequency effect is far less affected by practice (Bartram, unpublished manuscript): across eight blocks of trials, the frequency effect decreased from only 150 to $100 \mathrm{msec}$ instead of from about 350 to $100 \mathrm{msec}$, as in the present experiment. Such differences in the frequency effect for unpracticed Ss are difficult to account for in terms of response retrieval mechanisms. Instead, it can be argued that, while acquisition of knowledge of the response set does not in itself reduce the effect of frequency across practice, such knowledge can be used to overcome difficulties associated with processing "degraded" representations of rare objects. Identification threshold exposure studies have provided evidence to support the contention that perceptual processing is more difficult with line drawings than with photographs of objects (Fraisse \& Elkin, 1963; Ryan \& Schwartz, 1956). The increase in the frequency effect which occurs between Stages 1 and 3 can be explained in terms of the decay of information about the response set during the interpolated activity of Stage 2.

Rather than being a function of response retrieval process, then, the frequency effect may be a function of differences in the availability of information stored about the objects which are being named. It is proposed that this object information is used in the process of "perceptual identification" to form a description of the stimulus in terms of the type of object it depicts. Knowledge of the response set can be used to overcome the difficulties involved in processing "degraded" stimuli of LF objects by restricting the range of possible "hypotheses" concerning the identity of that stimulus object and thus decreasing the amount of information which needs to be retrieved. Thus, knowledge of the response set should facilitate the process of perceptual identification for objects regardless of whether this knowledge was gained initially from naming pictures or naming nonsense shapes, and this facilitatory effect should be greatest for LF objects represented by degraded stimuli.

In short, it has been suggested that practice with either picture or nonsense-shape stimuli has equivalent effects on reducing "object uncertainty." This reduction in uncertainty has a greater effect on naming latencies for LF objects than for HF ones, since information stored about LF objects is initially less available. Furthermore, these effects of object uncertainty are most prominent for LF objects when the stimuli used are "degraded" representations of the objects. The reduction in object uncertainty, as it results from knowledge of the response set rather than from knowledge of the stimuli, occurs during practice with the nonsense shapes and transfers to the subsequent picture-naming trials. Although object uncertainty at the beginning of Stage 2 is as low as at the end of Stage 1. "stimulus uncertainty" is high. This stimulus uncertainty did not interact with object frequency (as was seen, latencies for both LF and HF objects increased by $100 \mathrm{msec}$ on Block 8).

The frequency effect remaining after practice may be a function either of differences in name availability of the sort postulated by Oldfield or of long-term differences in the availability of information stored about common and rare objects. The present experiment does not allow a distinction to be drawn between these two possibilities.

\section{REFERENCES}

Attneave, F. Physical determinants of judged complexity of shapes. Journal of Experimental Psychology, 1957, 53, 221-227.

I raisse, W. F., \& Elkin, E. H. Étude génétique de l'influence des modes de présentation sur le seuil de reconnaissance d'objets familiers. Année Psychologie, 1963, 63, 1-12.

Oldfield, R. C. Things, words and the brain. Quarterly Journal of Experimental Psychoiogy, 1966, 18, 340-353.

Oldfield, R. C., \& Wingfield, A. The time it takes to name an object. Nature, 1964, 202, 1031-1032.

Oldfield, R. C., \& Wingfield, A. Response latencies in naming objects. Quarterly Journal of Experimental Psychology, 1965. 17, 273-281

Ryan, T. A., \& Schwartz, C. B. Speed of perception as a function of mode of presentation. American Journal of Psychology, 1956, 69, 60-69.

Thorndike, E. L., \& Lorge, I. The teacher's word book of 30,000 words. New York: Bureau of Publications, Teachers College, Columbia University, 1944.

Vanderplas, J. M., \& Garvin, E. A. The association value of random shapes. Journal of Experimental Psychology, 1959. $57,147-154$.

Wingfield, A. The identification and naming of objects Unpublished $\mathrm{PhD}$ thesis, Oxford University, 1966.

Wingfield, A. Effects of frequency on identification and naming of objects. American Journal of Psychology, 1968, 81. 226-234.

(Received for publication October 2, 1972; revision received October 16.1972.$)$ 